\title{
Erratum to: Investigating measurement equivalence of visual analogue scales and Likert-type scales in Internet-based personality questionnaires
}

\author{
Tim Kuhlmann ${ }^{1}$ - Michael Dantlgraber ${ }^{1}$ - Ulf-Dietrich Reips ${ }^{1}$
}

\section{Erratum to: Behav Res.}

DOI 10.3758/s13428-016-0850-x

1) In the Abstract, the penultimate sentence "The associations were largely identical, with the exception of an increase in explained variance when predicting age from the VAS version of Excitement Seeking $\left(\mathrm{B}_{10}=125.1, \Delta \mathrm{R}^{2}=.025\right)$." should be changed to "The associations were largely identical, with the exception of an increase in explained variance when predicting age from the VAS version of Excitement Seeking $\left(\mathrm{B}_{10}=1318.95, \Delta \mathrm{R}^{2}=.025\right)$."

2) On page 4, Statistical analyses, at the end of the second paragraph, the sentence that reads "To avoid type I-error inflation due to multiple testing, the significance level was set to." should be changed to "To avoid type I-error inflation due to multiple testing, the significance level was set to $\mathrm{p}<.01$."

The original article was corrected.

The online version of the original article can be found under doi:10.3758/ s13428-016-0850-x.

Tim Kuhlmann

tim.kuhlmann@uni-konstanz.de

1 Research Methods, Assessment and iScience, Department of Psychology, University of Konstanz, Box 31,

78457 Konstanz, Germany 